\title{
An Exploratory Investigation on Modelling Technologies to Flexible Loads Dispatching in A Smart Grid Environment
}

\author{
Kaiwen Zeng ${ }^{1, *}$, Fusheng $\mathrm{Li}^{2}$, Jianing Liu ${ }^{1}$, Haizhu Wang ${ }^{1}$, Dan $\operatorname{Lin}^{2}$, Weicong $\mathrm{Wu}^{2}$, and Tao $\mathrm{Yu}^{2}$ \\ ${ }^{1}$ Power Dispatching Control Center of Guangdong Power Grid Co., Ltd., Guangzhou 510600, Guangdong, China \\ ${ }^{2}$ School of Electric Power, South China University of Technology, Guangzhou 510641, Guangdong, China
}

\begin{abstract}
As the proportion of flexible load resources in smart grids continues to rise, resulting in increasingly complex grid structures, significant changes in grid characteristics, and increased risks to grid operation and control, it will be difficult to intelligently regulate the grid solely by relying on traditional resource regulation methods, and the dispatchable space for traditional resources will become smaller and smaller. To this end, this paper conducts an exploratory investigation on the modeling techniques for flexible loads participation in smart grid dispatching. First, a classification of the flexible loads involved in grid regulation is made. Secondly, according to the flexible load classification, the modeling techniques of different classes of flexible loads are reviewed and studied; then, the flexible load dispatching modes for different operating states and different control tasks, and under different control methods are discussed deeply. Moreover, the technological economics and feasibility of these different flexible load dispatching modes are compared. Finally, an outlook and conclusion are made.
\end{abstract}

\section{Introduction}

In recent years, with the concept of Energy Internet [1][2] proposed, and the rapid development of smart grids and integrated energy systems, the Global Energy Internet [3] will become the world's energy and power system development trend. At present, the proportion of new energy resources in the smart grid will continue to rise, resulting in an increasingly complex structure and significant changes in the characteristics of the grid, as well as the increased risk of operational control of the grid. In this context, it will be difficult to control the grid intelligently by relying only on traditional resource regulation methods, and the dispatchable space for traditional resources will become smaller and smaller [4]-[6]. Therefore, there is an urgent need to explore new dispatching technologies and methods to control grid resources, especially new energy resources. For example, these new dispatching ways include new grid dispatching operation modes that take into account generation uncertainty and load controllability [7], as well as research on the optimal dispatching method based on load controllability for main distribution coordination [8].

In China's Guangdong Province, for example, with the growing economic development and social demand for electricity in recent years, the Guangdong Power Grid has a significant increase in load peak-to-valley differential, load variation rate and the number of safe and stable sections [9], and its adjustable standby unit resources are also scarce, which brings more challenges to the company's daily safe dispatch operation control.
On the other hand, for the Guangdong Power Grid, with the development of the smart grid, more and more loads can be controlled within a certain range, and its flexible regulating capability changes the history of the load being regulated passively in one direction. The existing dispatch model for the Power Dispatching Control Center of Guangdong Power Grid Co., Ltd. has never considered flexible load participation in dispatch. Therefore, it is an urgent problem to explore the new power grid dispatching mode and study how to scientifically and reasonably regulate the massive multiflexible load, realize the coordination of mains and distribution, and improve the safe and stable operation of power grid [10]-[12]. Moreover, in order to adapt to the new situation of power grid development, to increase the means of power grid control, to solve the problem of Guangdong Power Grid peaking and frequency control difficulties, and to improve the safe operation of the power grid, it is necessary to actively explore new power grid dispatching operation mode, research on load controllability based on power grid dispatching control method, so as to effectively solve the current system peaking and frequency control difficulties, peak power generation side of the standby tension and other problems, and broaden the safety margin of the Guangdong Power Grid.

Currently, there are the following problems with load scheduling in China. First, the flexible load control approach at multiple time scales and multiple scheduling methods is rough, and the coordinated scheduling strategy for multiple types of controllable load is missing. Second, the main distribution network optimization

\footnotetext{
* Corresponding author: hust_kevin@foxmail.com
} 
separation and coordination scheduling mode is single, without considering the important role of flexible loads in the main distribution coordination. Third, the impact brought by flexible loads and its adjustable potential are not fully considered in peak frequency regulation and voltage control. Therefore, in the above context, this paper presents an overview study of modeling techniques for flexible loads participation in smart grid dispatch, including the following aspects. First, a classification of the flexible loads involved in grid regulation is made. Secondly, an exploration on modeling techniques for different classes of flexible loads based on the flexible load classification is conducted. Then, the flexible load dispatching modes under different operating states and control tasks and methods is deeply discussed, and the technical economy and feasibility of these different flexible load dispatching modes are compared. Finally, an outlook and conclusion are given.

\section{Flexible Load Classification and the Way to Match Flexible Loads to Smart Grid Functions and Demands}

Flexible loads [13] in a smart grid are those that can interact with the grid energetically and have flexible characteristics by actively participating in the control of the grid operation. Load flexibility is expressed as flexibility over a certain period of time [14]. Flexible loads include adjustable or transferable loads with demand flexibility, and loads with bidirectional adjustment capability, such as electric vehicles (EVs) [15], energy storage [16], distributed power supply [17], and micro-grid [18]. To better understand smart grid flexible loads, we illustrate them by the following three types.

\subsection{Classification according to load response/regulation mode}

According to load response/regulation mode, the flexible loads in smart grid can be divided into two categories, i.e., fast response loads and continuously regulated loads, and the definitions of each category and the main types of loads they contain are as follows.

In smart grid dispatching, fast response to load reflects the flexibility of the user's electricity consumption, and that the corresponding dispatch instructions on the grid side have fast response speed [19]. Management of rapid response loads can be used to alleviate peak load tensions, avoid or reduce costly spinning back-ups and investments in generating capacity needed to meet increased demand for electricity. Rapid response loads mainly include three types. The first one is EVs, which have a high response speed to the charging and discharging dispatching instructions from the grid. In terms of modeling of EVs, its demand capacity for a single EV is usually expressed by $S=(1-$ $S O C) C_{\max }$ [20], where $S$ is the charging demand when a single EV is connected to a charging station, $S O C$ is short for state of charge, indicating current battery charge state of the $\mathrm{EV}$, and $C_{\max }$ is the battery capacity. At this point, its charge/discharge power constraint can be expressed as $P_{\text {dis.max }} \leq P \leq P_{\text {char.max }}$, where $P_{\text {dis.max }}$ and $P_{\text {charmax }}$ are the maximum discharge power and maximum charge power that the battery can withstand in a safe operation state, respectively. In addition, its charging margin coefficient is expressed as $k_{\mathrm{coe}}=t_{\text {leave }} / t_{\mathrm{min}}$, where $t_{\text {leave }}$ and $t_{\min }$ is the time the EV leaves and the minimum charging time, respectively. Here, $k_{\text {coe }}$ can be used to measure the ability of EVs to participate in V2G, i.e., vehicle-to-grid.

Second, the interruptible loads, which mean that the customer signs an interruptible load agreement with the power company to reduce his demand for electricity during a fixed period of time during the grid's peak hours. Third, the incentive loads or transferable loads, which mean that consumption can be shifted from moments of higher electricity prices to moments of lower electricity prices out of consideration for the price of electricity. At this point, the grid needs to meet the power needs of the conventional load as well as those of the load aggregators in real time.

Continuously regulated load means that a load can receive periodic/non-periodic continuous control instructions from the grid based on its own operating characteristics or mode of operation, and has great potential value as a demand response resource in an electricity market environment. Continuously regulated loads include energy storage equipment and single-phase flexible loads. First, aiming at the optimization modeling of the energy storage equipment, its input/output transfer function can usually be expressed as $G_{\mathrm{B}}(s)=K_{\mathrm{B}} /\left(1+T_{\mathrm{B}} s\right)$. Here in the study of battery energy storage's participation in grid frequency regulation, we equate the battery energy storage system as a series of first-order inertial links and proportional links, which can visually and effectively describe the dynamic characteristics of its participation in grid frequency regulation behavior. Second, in terms of the modeling of electric water heater, its water temperature is expressed as $T_{t+1}^{\mathrm{hw}}=\left[\rho V_{t}^{\text {cold }}\left(T^{\text {cold }}-\right.\right.$ $\left.\left.T_{t}^{\mathrm{hw}}\right)+\rho V^{\mathrm{tan} k} T_{t}^{\mathrm{hw}}\right] /\left(\rho V^{\mathrm{tank}}\right)+h_{t}^{\mathrm{wh}} \Delta t /\left(\rho V^{\mathrm{tan} k} C_{\mathrm{w}}\right)$, where $T_{t}^{\mathrm{hw}}$ is the hot water temperature at time $t, \rho, V^{\text {tank }}$ and $C_{\mathrm{w}}$ are the density of the water, the volume of the tank and the specific heat capacity of the water, respectively, and $V_{t}^{\text {old }}$ is the volume of the cold water injected at time $t, T^{\text {cold }}$ is the temperature at which cold water is injected, and $h_{t}^{\mathrm{wh}}$ is the heating power at time $t$. At this point, the electric water storage tank also needs to meet the upper power limit constraint and temperature deviation constraint expressed as $0 \leq P_{t}^{\mathrm{wh}} \leq P_{\max }^{\mathrm{wh}}$ and $\Delta T_{\text {min }}^{\mathrm{hw}} \leq \Delta T_{t}^{\mathrm{hw}} \leq \Delta T_{\max }^{\mathrm{hw}}$.

\subsection{Classification according to energy interaction characteristics}

Flexible loads are divided into two-way interactive flexible loads and one-way flexible loads according to their energy interaction. Two-way interactive flexible loads are typical for EVs and energy storage, and oneway flexible loads are typical for demand response 
resources such as heaters, air conditioners, and refrigerators in residential loads.

Here, two-way interactive flexible loads include EVs and energy storage devices. For the modeling of EVs, the mainstream lithium-ion battery is usually used as an example to model EV charging and discharging. Since lithium-ion batteries are in the pre-charge phase and the duration of charging in the constant-voltage segment is short, the charging process for EVs is generally considered to be constant. Power Charge. For modeling convenience, it can be approximated that the power is constant during charging and discharging of EVs. At the present stage, the modeling of battery energy storage system mainly focuses on the equivalence of the charging and discharging circuits of the battery system, and then determine the equivalence parameters through experiments, so as to complete the construction of the battery system model.

In addition, the unidirectional flexible load modeling including electric water heater modeling, general heating/refrigeration air conditioning modeling, and refrigerator/freezer load modeling.

\subsection{Classification according to different management styles}

According to different management methods, flexible loads in the smart grid are classified into interruptible and incentive loads. For interruptible load, when a customer enters into an interruptible load agreement with an electric utility, it reduces its electricity demand for a fixed period of time during the peak hours of the grid. The interruptible load is assumed to have two states: on and off. Once this device is turned on, it runs at a certain power level. It has to run for a certain amount of time to complete a certain amount of electricity within a certain time frame. In the case of incentive load, consumption behavior can be shifted from moments of higher electricity prices to moments of lower electricity prices out of consideration for the price of electricity. The grid needs to meet the power needs of the conventional load as well as the load aggregators in real time. Therefore, the total power supply to the distribution network and the total power required by the load aggregators can be expressed by the following formula, i.e., $Q_{\text {total }}(t)=Q_{\text {load }}(t)+Q_{\text {sum }}(t) \quad$ and $\quad Q_{\text {sum }}(t)=\sum_{i=1}^{N} Q_{i}(t) \quad$, where $t \in T, Q_{\text {total }}(t)$ is the total amount of electricity supplied by the grid in time period $t, Q_{\text {load }}(t)$ is the total electricity demand of the conventional load in period $t$, $Q_{\text {sum }}(t)$ is the sum of the power purchased by the load aggregator in time period $t$, and $N$ is the number of load aggregators in the area under the jurisdiction of the grid. Here, a common dynamic pricing model for electricity is shown as $p(t)=\delta(t) \cdot Q_{\text {total }}(t)$, where $p(t)$ is the electricity price for the $t$ th dispatch period, and $\delta(t)$ is the electricity price coefficient, characterizing the extent to which the tariff is affected by $Q_{\text {total }}(t)$, and such coefficient is shown as $p_{\max } / Q_{\max }$, where $p_{\max }$ is the upper limit of the electricity price set by the distribution network, which can be set based on government policy or actual operating experience of the network, and $Q_{\max }$ is the power supply limit of the distribution network. The classification of flexible loads facilitates their modeling and regulation studies and facilitates the application of different characteristics of flexible loads in different scenarios of power systems.

Overall, the classification of flexible loads in the smart grid is shown in Fig. 1.

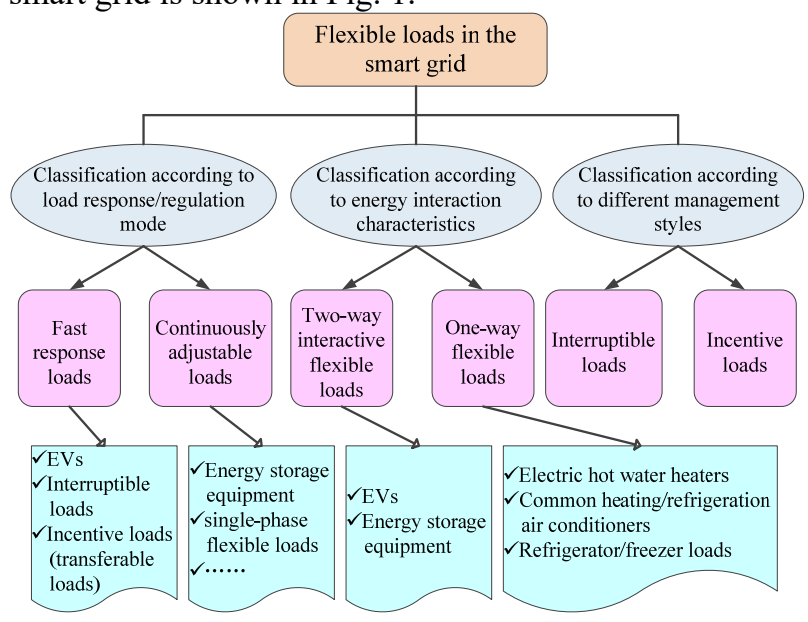

Fig. 1. Classification of flexible loads in the smart grid.

\subsection{Ways to match flexible loads to smart grid functions and demands}

Flexible loads are modeled by agents to form load clusters of a certain size, and when this cluster reaches a certain magnitude, it has the ability to participate in grid dispatch, and by properly arranging this load cluster can have an impact on the function and demand of the grid. In addition, the flexible load cluster can ultimately be made to produce the following benefits by forming an interaction with the grid through the above mentioned agents and by participating in the grid dispatch operation based on the mode of flexible load in different power system operating states, as described below.

Firstly, aiming at the matching approach for normal operating conditions, the benefits include three aspects. The first aspect is the benefits of cutting peak and filling valley of the smart grid. At this point, the storage batteries and EVs can be charged when the load is low and discharged during the peak period, which can effectively relieve the pressure of peak adjustment during the peak period of the power grid, realize the peak reduction and valley filling of the load curve, and improve the load rate and the efficiency of equipment use. The second aspect is to provide ancillary services to the smart grid system. In the conventional system, the power plant provides the peaking, frequency regulation and various other resources. This is also possible if the flexible load is controlled. The last aspect is the benefits obtained from optimization of power flow distribution. At this point, due to the spatial dispersion of the flexible load, controlling the electricity consumption behavior of the flexible load can influence the tidal current distribution of the power system, which can be used to reduce system network losses and improve the voltage quality of the system. 
Secondly, aiming at the matching approach for emergency and recovery states, the benefits are obtained from two aspects. The first one is to optimize power grid flow from active power dispatching. Flexible load optimizes the distribution network's tidal current distribution by providing active work, and in the case of bi-directional interactive flexible load, the distribution network can be discharged in case of a safety and stability failure to improve the current operating state of the distribution network. The second one is to improve voltage stability via reactive power optimization. At this point, the reactive power support to the power grid is realized through the switching control of various types of flexible loads, which improves the transient voltage stability margin of the distribution network, improves the voltage quality and transient voltage stability, and restores the transient voltage stability of the power system in case of emergency as soon as possible.

On the whole, the way in which flexible loads are matched to grid functions and demands is shown in Fig. 2 below.

\section{Functions and demands of the smart grid}

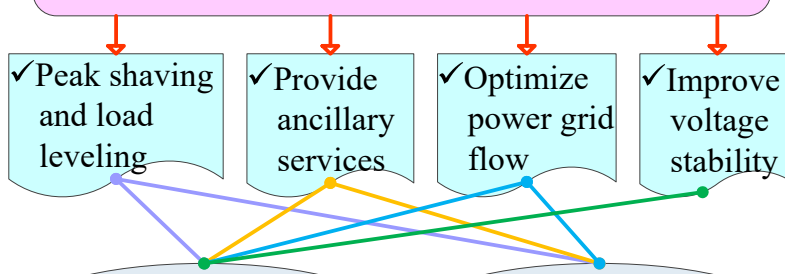

Two-way interactive

\begin{tabular}{|l|l|}
\hline flexible loads & One-way flexible loads \\
$\checkmark$ Energy & $\checkmark$ Electric hot water heaters \\
storage & $\checkmark$ Common heating/refrigeration \\
equipment & air conditioners \\
$\checkmark \ldots \ldots .$. & $\checkmark$ Refrigerator/freezer loads \\
Flexible loads in smart grid
\end{tabular}

Fig. 2. Illustration of the ways to match flexible loads to smart grid functions and demands.

\section{Discussion on Dispatching Models Considering Flexible Loads in Smart Grid}

\subsection{Flexible load dispatching mode for different operating states}

Flexible load dispatching for smart grids under different operating states consists of two modes, i.e., dispatching modes for considering flexible load in normal and abnormal states. For the former, i.e., under normal conditions, on the one hand, through multi-level coordination and grade-by-level refinement at different time scales, the aim is to gradually reduce the impact of flexible load uncertainty on grid operation. On the other hand, through collaborative dispatching on the generation side and the load side, the aim is to ultimately achieve optimal matching of source and load resources.
In general, in this dispatching model, the dispatching strategy can be decomposed into two phases before and during the day. For each phase, there needs to be coordination between the grid and the equipment side, taking into account the impact of the distribution network dispatching operation on the main network, thus taking advantage of the complementary resources of the source and the destination. For the latter, i.e., under abnormal conditions, the smart grid mode of flexible load participation can be classified as either a fault state or a maintenance state. In a fault condition, first, the total amount of load to be cut is determined based on demand. Then, the total amount to be cut is allocated to each execution station according to the precise load-cutting control strategy of the distribution network, so as to effectively respond to grid faults.

In the maintenance state, it is necessary to make the maintenance plan model a two-layer, multi-constrained optimization model through the participation of flexible load. In addition, the involvement of flexible load makes the maintenance plan decision more flexible, and flexible load can adjust the electricity consumption plan according to the maintenance plan. Avoiding conflicts with maintenance schedules further reduces system maintenance costs and the probability of failure during maintenance.

On the whole, the flexible load dispatching mode for different operating states can be shown as illustrated in Fig. 3.

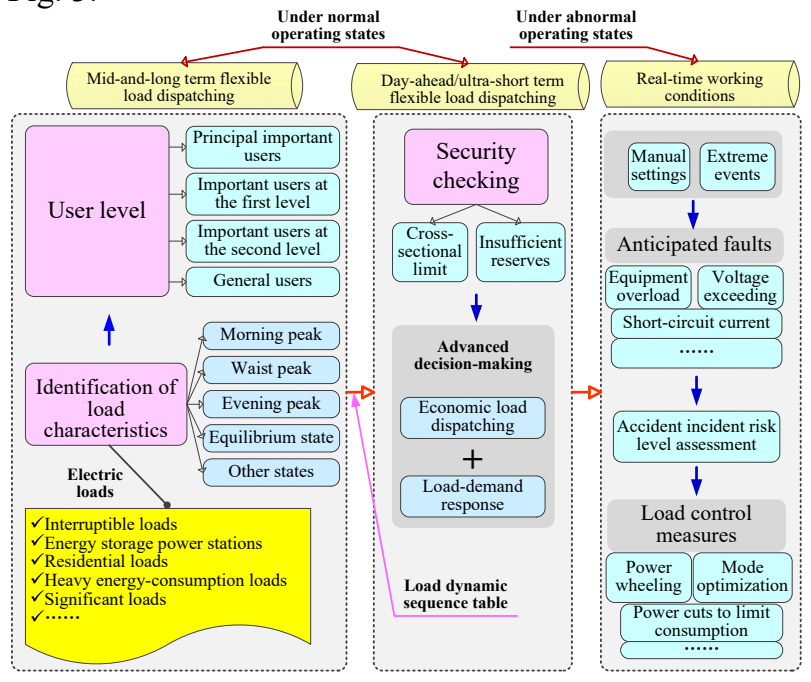

Fig. 3. Illustration of the flexible load dispatching mode for different operating states.

\subsection{Flexible load dispatching mode for different control tasks}

For flexible load dispatching under different control tasks in the smart grid, there are two types of load control models, namely flexible load dispatch model in active dispatch and flexible load dispatch model in reactive dispatch, which are discussed as follows.

First, for the study of flexible load dispatching models in active power dispatch, the first step is to establish an active dispatch model that considers flexible loads. The objective function of this model can be 
expressed as $\min C\left(\boldsymbol{P}_{\mathrm{G}}\right)=\min \sum_{t=1}^{T} \sum_{i \in S_{\mathrm{G}}} f\left(P_{\mathrm{G} i, t}\right)$, where $f\left(P_{\mathrm{G} i, t}\right)$ is shown as $0.5 a_{i} P_{\mathrm{G} i, t}^{2}+b_{i} P_{\mathrm{G} i, t}$. Here, this objective function needs to satisfy six sets of constraints. The first one is system power balance constraint, which is presented as $\quad-\sum_{j \in \mathrm{S}} B_{i j} \theta_{j, t}=P_{\mathrm{G} i, t}+P_{\mathrm{C} i, t}+P_{\mathrm{H} i, t}-P_{\mathrm{L} i, t}-P_{\mathrm{FL} i, t}^{(1)}$, where $P_{\mathrm{G} i, t}$ is the output power of conventional generators, $P_{\mathrm{C} i, t}$ is the output power of pumped storage units, $P_{\mathrm{H} i t,}$ is the output power of nuclear generators, $P_{\mathrm{L} i, t}$ is the electric loads of conventional consumers, and $P_{\mathrm{FL} i, t}^{(1)}$ is the transferable flexible loads. Obviously, when a power system operates, it must ensure the above power balance. The second one is unit climbing constraint, which is shown as $-P_{G i}^{d} \leq P_{G i, t}-P_{G i, t-1} \leq P_{G i}^{u}$, where $P_{G i, t}$ and $P_{G i, t-1}$ are the output power of the unit at this moment and the previous moment, respectively. According to this constraint, the climbing rate of the unit must satisfy the minimum and maximum climbing constraints during the two adjacent periods. This third one is pumped storage constraint, which can be described as $\sum_{t=1}^{T} P_{c i, t}=0$. Based on this constraint, pumped storage generators generate electricity and transmit power to the grid during peak periods when electricity prices are high, while pumping water during periods of low consumption consumes electricity. Over the course of a day, the sum of the generation and consumption of electricity from the pumped storage generators can be considered to be zero. The fourth one is nuclear power constraint, which can be presented as $P_{h i, t}=$ cons $\tan t$, where $t=1,2, \cdots, T$. Here, nuclear power units produce stable power and their output can be considered constant over time. The fifth one is line safety constraint, which can be described as $-P_{i j}^{\max } \leq B_{i j}\left(\theta_{i, t}-\theta_{j, t}\right) \leq P_{i j}^{\max } \quad, \quad$ where $\quad l=1,2, \ldots, L \quad$ and $t=1,2, \cdots, T$. Here, the line safety constraint ensures that the line transmission power does not exceed the limit, ensuring the safe and stable operation of the grid. The last one is transferable load constraint. Here, consider the flexible load $P_{\mathrm{FL} i, t}^{(1)}$ at node $i$ in time period $t$ as a transferable load that satisfies the total load for the day unchanged, i.e., $\sum_{t=1}^{T} P_{\mathrm{FL} i, t}^{(1)}=T P_{\mathrm{FL} i}$, where $T P_{\mathrm{FL} i}$ is the total transferable flexible load for one day at the $i$ th node.

Flexible load-based coordinated dispatching between active and reactive power refers to the introduction of reactive power dispatch, which is neglected in traditional power systems, into microgrids dispatching and optimization. This differs from the traditional decoupling of active and reactive power for optimal dispatch separately, but couples the interactive active and reactive power between the microgrid and the large grid. The restriction on the power factor of a large grid on the microgrid's interaction with it is done by adding a reactive rewards and penalties charging module to improve the power factor of the microgrid. To motivate users to be self-sufficient in reactive power, the grid can acquire excess reactive power from the users. The microgrid is rewarded when the reactive power ingested by the microgrid is below the permissible limit, and the microgrid is penalized when the reactive power ingested by the microgrid is above the limit. Based on this, the basic model of the flexible load scheduling model in reactive power optimization can be described as follows. The model is described as $C_{\mathrm{b}}=\sum_{t=1}^{T} C_{\mathrm{pf}}\left(Q_{\mathrm{d}, t}-Q_{\mathrm{d} 0, t}\right) \lambda_{t}$, where $C_{\mathrm{b}}$ is the cost of the large grid penalty to the microgrid at time $t$ (with unit $\$$ ), $C_{\mathrm{pf}}$ is the penalty coefficient (with unit $\$ /$ Var), $Q_{\mathrm{d} 0, t}$ is the maximum reactive power intake specified by the larger grid (with unit Var), and $\lambda_{t}$ is the total time of dispatching.

\subsection{Flexible load dispatching mode under different control methods}

As the rapid development of smart grid and energy Internet, an increasing variety of complex flexible loads are involved in load control and dispatching throughout large power systems and integrated energy systems. Taking Guangdong Province in China as an example, Guangdong Province now has a certain scale of flexible load access and distributed power supplies such as photovoltaic power generation, plant waste heat generation, and combined cooling, heating, and power generation. The Southern Power Grid has planned to build a large number of charging stations in Guangdong province, especially in Shenzhen, which is the first city to promote the use of electric buses on a large scale. Guangdong Province is carrying out different types of energy storage planning and layout, so it can be predicted that in the next two years, the province's distributed power supply, EVs, flexible loads, and all types of energy storage will grow rapidly, and eventually form a new form of power system that interconnects and interacts source, grid, loads and energy storage. In addition, there are many industrial parks in Guangdong province, and the current economic form is not very prosperous, so large users of power enterprises have an incentive to participate in demand-side response to reduce production and operating costs and enhance the market competitiveness of their products. Generally speaking, the overall load in Guangdong Province is large and diverse, and has a certain degree of regulation and control, and there is also a certain degree of complementarity between different types of loads. Therefore, the implementation of flexible dispatching in Guangdong Province is characterized by large space for development and extensive benefits.

The above-mentioned flexible load dispatching mode in Guangdong Province or other provinces contains three specific control modes, i.e., flexible load dispatching mode under direct control, flexible load dispatching based on microgrids, and flexible load dispatching mode for virtual power plants (VPPs). These three modes are discussed as follows.

First, we discuss the direct control type of flexible load dispatching in smart grid. Obviously, in traditional power grids, each power plant is generally managed and controlled by the grid's dispatch center. This type of direct control is also a possible form of management for 
load-side equipment such as distributed power, energy storage and flexible loads. This is illustrated in Fig. 4 below.

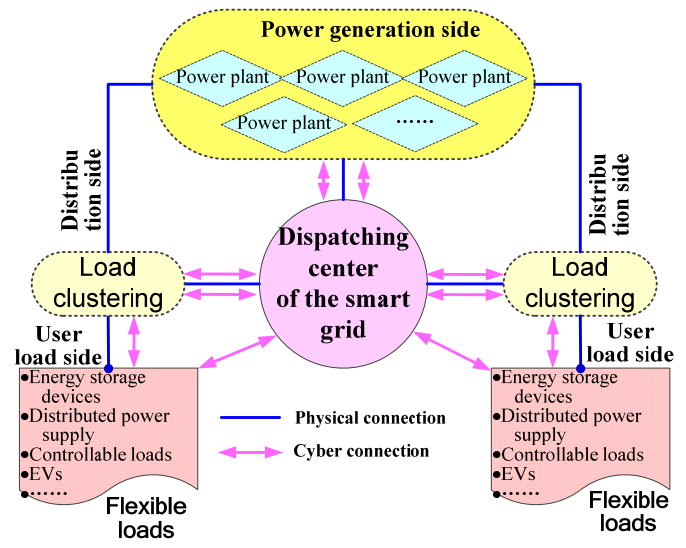

Fig. 4. Illustration of the flexible load dispatching of smart grid under direct control method.

As illustrated in Fig. 4, in this direct control mode, each load-side device maintains its original topology electrically, but is connected to the dispatching center of the power grid through a dedicated communication network. At this point, each plant and load-side device uploads its own operating parameters to the dispatching center, which optimizes the source and load information of the whole network and sends the dispatching information to each plant and user-side device via a dedicated communication network. The advantages of load scheduling under direct control include the following three aspects. Firstly, all information is summarized in the dispatching center, so that the optimized strategy can obtain the global optimal solution based on complete information, achieve better dispatching effect, reduce overall energy consumption cost, and maximize social benefit. Secondly, the dispatching center has the ability to directly monitor and control terminal devices, which allows for more flexible and precise dispatching execution; due to the reduction of intermediate levels, the control delay for terminals is greatly shortened when the network is smooth, thus enabling the dispatching center to carry out control policies requiring high real-time performance. Lastly, in direct control mode, the original electrical topology of the network can be kept unchanged, and only modifications in communication are required, making it easy to modify existing equipment. The disadvantages of direct control are reflected in both low necessity and poor feasibility. Specifically, the reason for the low necessity is that the individual capacity of load-side distributed devices is often small, and the individual devices can be used for both economic dispatching and The impact on the grid in stabilization control is all minimal. In this case, direct control of every load-side device by the grid dispatch center is unnecessary.

In addition, the infeasibility of this direct control method is reflected in three aspects: high economic cost, poor security, and high difficulty in implementing the dispatching algorithm. Firstly, the dispatching center must be equipped with a communication system for each device and a dedicated communication network must be built for direct control of each device. In the context of load-side devices tending to be massive, the construction cost of the communication system alone is unbearable. Secondly, the reason for the poor security is that it is difficult to determine the network security status of loadside devices in the direct control mode. On the one hand, since these devices are directly connected to the dispatch center of the grid, they are logically equal to the power plant, for which reason these load-side devices should be connected to the control area (security zone I), but the cost of the vertical encryption device thus generated is unacceptable. On the other hand, the negligible impact on the grid due to the very small capacity of individual distributed devices may allow consideration of reducing their security level to reduce security investment, but this creates a risk of data spoofing for both parties, and the risk to the grid is very high due to the fact that the grid dispatch center directly controls the end devices and must assume responsibility for security on the user side. Lastly, when a large number of load-side devices are directly controlled by the dispatching center, the state of each device becomes a decision variable for optimal dispatching, which is orders of magnitude different from the original generation-side variables, and the resulting dimensional disaster problem not only tests the dispatching center's hardware capabilities and optimization algorithms, but also makes the problem unsolvable.

Second, we discuss flexible load dispatching mode based on microgrid or microgrids in a smart grid. Through the microgrid, new energy power generation equipment is no longer connected directly to the power grid alone, but is connected to the local load, power electronic power conversion device, energy storage system, etc. to form an integrated power generation control system by dispatching the microgrid control strategy and then connected to the power grid. By coordinating and controlling the new energy (wind power, solar power, etc.) and other types of power supply, energy storage system, load and distribution equipment in a region, the micro-grid enables new energy to be produced and consumed locally. Microgrids allow new energy to be utilized in a variety of ways, either in off-grid mode to power local loads or as a node in the grid, which is a powerful tool for adjusting the energy structure and promoting the development of new energy. Microgrids are characterized by the fact that they physically form a localized mini-grid and the grid dispatch center is no longer interested in the specific devices within the microgrid. Instead, it is concerned with the line of communication between the microgrid and the larger grid. In this model, scheduling within the microgrid is performed by the microgrid's own control center, which is connected to the grid via a network channel. The dispatch center is connected and receives dispatch from the grid. The advantages of this flexible load dispatching mode is reflected as follows. First, as a physically connected network, the microgrid is well connected internally, and local dispatch management is easy to implement and can be very effective. Second, as micro-networks integrate various devices within their respective areas, the micro-network control center is connected to the grid dispatch center, reducing the 
pressure on the grid dispatch center and reducing the investment cost for communication system and network security. However, such mode also has some disadvantages during flexible load dispatching in a smart grid. First, while microgrids can fulfill the grid's need for cluster management of distributed devices, the architecture of microgrids limits the distributed geographic range of devices, it can only form a physical mini-grid of devices in a small area before connecting them to the larger grid. This reduces the scale and flexibility of a microgrid to a large-scale, multiownership, distributed device architecture for gridconnected management systems. Second, for existing power users, the construction of a microgrid requires the purchase of new equipment and the renovation of the existing network, which requires a certain amount of investment and may affect users' willingness to participate in load dispatching. Third, since the participating users have been bound as a whole since the construction of the microgrid, the users within the zone can only receive services from one power operator, which prevents the users from freely choosing the operator and makes it difficult for other operators to enter, reducing market competition and vitality.

Third, we discuss the flexible load dispatching mode based on VPPs in a smart grid. At this point, the main advantage of a VPP over a microgrid is that it does not have strict requirements on the geographic location and ownership of equipment within the network, and can achieve unified scheduling and control of a large range of large-scale distributed equipment. The VPP, on the other hand, uses advanced communication technology to aggregate and control distributed power supplies, mainly through its internal control center. The advantages of such flexible load dispatching mode are reflected as follows. First, any distributed device that is connected to the grid and has a communication connection to the control center can be included in the virtual power plant, regardless of geographic location and electrical distance, integrating a large number of user-side resources in a widely distributed area and allowing access to existing users without modifying the network. Second, the connection between virtual power plant and power grid is relatively tighter and more reliable than microgrid, which is connected to the power grid through a wide distribution of contact points, generally does not become a closed system operating independently, and has been with the main network for energy flow, information flow, money flow exchange, showing the characteristics of an open system. Third, in the power market environment, as virtual power plants are much larger than micro-grids, they are large enough to compete with traditional power producers and can adapt to the power market. The rules of the electricity market will be applied to obtain more benefits for the distributed customers participating in the virtual power plant. At the same time, since the relationship between the customer and the virtual power plant is only virtual, the customer is free to choose a suitable operator. However, such dispatching mode also has some disadvantages. First, compared to microgrids, virtual power plants, because the concept of logical and physical independence is not the same, will involve a larger area and more complex scheduling problems, which will require a very high management algorithm for virtual power plants. Second, terminal equipment in the same region may not belong to the same virtual power plant, increasing operational constraints and decreasing internal scheduling flexibility, thus reducing the effectiveness of local energy consumption compared to microgrids. Third, the virtual plant management center may compromise the maximization of social benefits with the goal of maximizing its own profits.

\subsection{Technological economics and feasibility of different flexible load dispatching modes}

The technological economics of the different flexible load dispatching modes can be measured by construction cost indicators, and the technical feasibility of the different modes can be measured by indicators of access capacity, control difficulty, whether the network needs to be modified, and control flexibility, as shown in Table 1 below.

Table 1. Technological economics and feasibility of different flexible load dispatching modes.

\begin{tabular}{cccccc}
\hline $\begin{array}{c}\text { Dispatching } \\
\text { mode }\end{array}$ & $\begin{array}{c}\text { construction } \\
\text { cost }\end{array}$ & $\begin{array}{c}\text { Access } \\
\text { capacity }\end{array}$ & $\begin{array}{c}\text { Control } \\
\text { difficulty }\end{array}$ & $\begin{array}{c}\text { Whether grid } \\
\text { reconstruction } \\
\text { is needed }\end{array}$ & $\begin{array}{c}\text { Control } \\
\text { flexibility }\end{array}$ \\
\hline $\begin{array}{c}\text { Direct } \\
\text { control }\end{array}$ & High & Low & High & No & High \\
\hline $\begin{array}{c}\text { Based on } \\
\text { microgrid }\end{array}$ & Moderate & Moderate Moderate & Yes & Moderate \\
\hline $\begin{array}{c}\text { Based on } \\
\text { VPP }\end{array}$ & Low & High & High & No & Low \\
\hline
\end{tabular}

As presented in Table 1, the direct control mode provides direct communication control of specific loads, which allows for good control flexibility. However, due to the huge amount of load, it is very difficult to control the load using direct control as the only control method. Although there is no need to transform the power network, the communication network construction cost of direct control is very high under the consideration of safety, which brings high construction cost to direct control and also leads to the inability of direct control to access the excessive number of loads and limits its access capacity.

A microgrid is effectively a local mini-grid with its own network structure and dispatch system. Since the controlled devices are all local, their control flexibility is relatively high and their control difficulty is reduced. The control pressure on the grid is also reduced because only the microgrid clusters need to be controlled. However, since a microgrid is a physical network, its composition requires corresponding network modifications, resulting in higher construction costs and a lower cost of ownership. It is not possible to build the microgrid very large.

VPPs communicate with various load-side devices and do not require physical networking of member devices. Therefore, the construction cost is low, and a large number of devices can be connected easily and quickly to form a large-capacity virtual power plant. However, since the member devices are not entirely 
local, the virtual power plant has more internal control constraints, reduced flexibility and increased control difficulties.

\section{Prospect and Conclusion}

\subsection{Prospect}

In order to adapt to the new situation of power grid development, it is necessary to increase the means of power grid regulation and control, solve the difficulties of power grid peaking and frequency regulation, and improve the safe operation of power grid. Therefore, it is still necessary to further study new grid dispatching operation modes in the future, especially the new grid load dispatching mode with flexible load participation. At the dispatching level, the coordinated control strategy on how adjustable load resources and traditional dispatching resources can participate in grid peaking, frequency regulation and even accident disposal scenarios during the day and the day before should be further studied [9], [15]-[18]. By researching the grid dispatching control method based on load controllability, it is possible to effectively solve the current difficulties of system peak control and frequency adjustment, and the power generation side's standby tension in the peak period, and widen the safety margin of the power grid.

\subsection{Conclusion}

This paper focuses on a new grid dispatch operation model that considers generation uncertainty and load controllability. Firstly, the different types of flexible load are classified into two-way interactive flexible load and one-way flexible load according to energy interaction, and interruptible load and incentive load according to different management methods. Secondly, the different types of flexible load are matched to the functions and demands of the grid for normal and emergency states and recovery states. The flexible load dispatching modes and their advantages and disadvantages under the three control modes of direct control, microgrid and virtual power plant are studied. Finally, the techno-economic and feasibility of the different modes are analyzed and prospects are presented.

\section{Acknowledgment}

This work was supported by the Science and Technology Project of China Southern Power Grid Company Ltd., under Grant GDKJXM20180576.

\section{References}

1. L.F. Cheng, T. Yu, X.S. Zhang, L.F. Yin, and K.Q. $\mathrm{Qu}$, "Cyber-physical-social systems based smart energy robotic dispatcher and its knowledge automation: framework, techniques and challenges," Proceedings of the CSEE, vol. 38, no. 1, pp. 25-40, Jan. 2018.
2. J. Rifkin, The third industrial revolution: how lateral power is transforming energy, the economy, and the world, Palgrave MacMillan, New York, USA, pp. 24-71, 2011.

3. Z.Y. Liu, Global energy internet, China Electric Power Press, Beijing, China, pp. 1-10, Feb. 2015.

4. L.F. Cheng, T. Yu, X.S. Zhang, and L.F. Yin, "Machine learning for energy and electric power systems: state of the art and prospects," Autom. Electr. Power Syst., vol. 43, no. 1, pp. 15-31, Jan. 2019.

5. T. Yu, L.F. Cheng, and X.S. Zhang, "The weaklycentralized Web-of-Cells based on cyber-physicalsocial systems integration and group machine learning: Theoretical investigations and key scientific issues analysis," Sci. Sin. Tech., vol. 49, pp. 1541-1569, 2019.

6. D.S. Callaway and I.A. Hiskens, "Achieving controllability of electric loads," Proc. IEEE, vol. 99, no. 1, pp. 184-199, 2011.

7. A. Botterud, Z. Zhou, J. Wang, et al., "Demand dispatch and probabilistic wind power forecasting in unit commitment and economic dispatch: a case study of Illinois," IEEE Trans. Sustain. Energy, vol. 4, no. 1, pp. 250-261, 2013.

8. L.F. Cheng and T. Yu, "Game-theoretic approaches applied to transactions in the open and ever-growing electricity markets from the perspective of power demand response: An overview," IEEE Access, vol. 7, no. 1, pp. 25727-25762, Mar. 2019.

9. B.J. Wen, B. Yu, Y.H. Bai, T.T. Ma, and C.X. Guo, "Research of the overall solution of next generation dispatching automation support system for Guangdong Power Grid," Southern Power System Technology, vol. 5, no. 1, pp. 18-21, 2011.

10. L.F. Cheng and T. Yu, "Typical scenario analysis of equilibrium stability of multi-group asymmetric evolutionary games in the open and ever-growing electricity market," Proceedings of the CSEE, vol. 38, no. 19, pp. 5687-5703, Oct. 2018.

11. A. Khodaei, S.M. Shahidehpour, and S. Bahramirad, "SCUC with hourly demand response considering intertemporal load characteristics," IEEE Trans. Smart Grid, vol. 2, no. 3, pp. 564-571, 2011.

12. K.Y. Huang, H.C. Chin, and Y.C. Huang, "A model reference adaptive control strategy for interruptible load management," IEEE Trans. Power Syst., vol. 19, no. 1, pp. 683-689, 2004.

13. L. Zhang, T. Zhang, F.Z. Wang, and X. Qi, "Ultrashort-term forecasting method based on response characteristics of flexible load," Power System Protection and Control, vol. 47, no. 9, pp. 27-34, 2019.

14. S.C. Yang, J.T. Liu, J.G. Yao, H.F. Ding, K. Wang, and Y.P. Li, "Model and strategy for multi-time scale coordinated flexible load interactive scheduling," Proceedings of the CSEE, vol. 34, no. 22, pp. 3664-3673, Aug. 2014. 
15. X.F. Wang, C.C. Shao, X.L. Wang, and C. Du, "Survey of electric vehicle charging load and dispatch control strategies," Proceedings of the CSEE, vol. 33, no. 1, pp. 1-10, Jan. 2013.

16. Y. Sha, X.Y. Qiu, X.J. Ning, and X. Han, "Multiobjective optimization of active distribution network by coordinating energy storage system and flexible load," Power System Technology, vol. 40, no. 5, pp. 1394-1399, May 2016.

17. X.Y. Duan, Z.C. Hu, Y.H. Song, and H.M. Chen, "Two-stage optimization of distributed network operation strategy with electric vehicle and distributed energy," Journal of Global Energy Interconnection, vol. 1, no. 1, pp. 87-95, Jan. 2018.

18. Z. Zhang, J.X. Wang, and X.Y. Cao, “An energy management method of island microgrid based on load classification and scheduling," Autom. Electr. Power Syst., vol. 39, no. 15, pp. 17-23+109, Aug. 2015.

19. L.F. Cheng and T. Yu, "Nash equilibrium-based asymptotic stability analysis of multi-group asymmetric evolutionary games in typical scenario of electricity market," IEEE Access, vol. 6, no. 1, pp. 32064-32086, Dec. 2018.

20. Z.N. Pan, X.S. Zhang, T. Yu, and D.Z. Wang, "Hierarchical real-time optimized dispatching for large-scale clusters of electric vehicles," Autom. Electr. Power Syst., vol. 41, no. 16, pp. 96-104, Aug. 2017. 